\title{
ON THE PROJECTIVITY AND FLATNESS OF SOME GROUP MODULES
}

\author{
GERHARD RACHER \\ Universität Salzburg \\ Hellbrunner Straße 34, A-5020 Salzburg, Austria \\ E-mail: gerhard.racher@sbg.ac.at
}

\begin{abstract}
In the sequel of the work of H. G. Dales and M. E. Polyakov we give a few more examples of modules over the Banach algebra $L^{1}(G)$ whose projectivity resp. flatness implies the compactness resp. amenability of the locally compact group $G$.
\end{abstract}

Let $L^{1}(G)$ be the $L^{1}$-algebra associated with a left invariant Haar measure on the locally compact group $G$. In the sequel of the work of H. G. Dales and M. E. Polyakov, D-P], we will give a few more examples supporting Helemskii's philosophy on the relation between the projectivity of $L^{1}(G)$-modules and the compactness of $G$ on the one hand, and between the flatness of $L^{1}(G)$-modules and the amenability of $G$ on the other; see for instance [He1, p. 238], or [He1, IV. Theorem 5.13, p. 190] and [He1, VII. Theorem 2.35 , p. 260].

If $A$ is an abstract Banach algebra and $A_{+}=A \oplus \mathbb{C}$ its unitization, $L_{a}$ will denote the operator of left multiplication by $a \in A$ on either $A$ or $A_{+}$. A Banach left $A$-module $X$ will always be contractive such that the action $\pi: A \widehat{\otimes} X \rightarrow X, \pi(a \otimes x)=a x$, is a linear contraction; $\widehat{\otimes}$ denotes the projective tensor product of Banach spaces, and $\mathcal{L}$ the space of all bounded linear mappings. For any Banach left $A$-module $X$, its dual Banach space, $X^{*}$, becomes a Banach right $A$-module by defining $\left\langle x, x^{*} a\right\rangle=\left\langle a x, x^{*}\right\rangle$, for $x \in X, x^{*} \in X^{*}, a \in A$. We shall always use the canonical isometrical isomorphism $(A \widehat{\otimes} X)^{*}=\mathcal{L}\left(A, X^{*}\right)$.

A Banach left $A$-module $X$ is called essential if the linear span of the products $a x$ ( $a \in A, x \in X)$ is dense in $X$. In case $A=L^{1}(G)$, every essential Banach left $L^{1}(G)$ module is a Banach $G$-module such that for any $x \in X$ the map $s \mapsto s x$ is continuous from $G$ into $X$ and satisfies $\|s x\|=\|x\|$ for all $s \in G$. Conversely, every Banach $G$-module

2010 Mathematics Subject Classification: 43A20, 46H25, 46M10.

Key words and phrases: projective $L^{1}(G)$-module, flat $L^{1}(G)$-module, amenable group. The paper is in final form and no version of it will be published elsewhere. 
is an essential Banach $L^{1}(G)$-module. Left translation by $s \in G$ will be denoted by $L_{s} f(t)=f\left(s^{-1} t\right)$, for any function $f$ on $G$.

1. Projectivity over $L^{1}(G)$. Instead of giving the original definition, cf. $\mathrm{D}-\mathrm{P}$, Definition 1.1, p. 392], we shall use the following criterion, [D-P, Proposition 1.2, p. 392].

1.1. Criterion. Let $A$ be a Banach algebra and $X$ be a Banach left $A$-module. $X$ is projective if and only if there is a bounded linear map $\rho$ such that $\pi \circ \rho=1_{X}$ and $\rho(a x)=\left(L_{a} \widehat{\otimes} 1_{X}\right)(\rho x)$, for $x \in X, a \in A$ :

$$
X \stackrel{\rho}{\longrightarrow} A_{+} \widehat{\otimes} X \stackrel{\pi}{\longrightarrow} X .
$$

$X$ is called $c$-projective, for some constant $c>0$, if there is such a $\rho$ of norm $\|\rho\| \leqslant c$, cf. [W, Proposition 2.8, p. 158]. If $X$ is essential, $A_{+}$may be replaced by $A$.

1.2. Let $A$ be $L^{1}(G)$. If $G$ is compact, then every essential Banach left $L^{1}(G)$-module $X$ is 1-projective. Denoting the continuous contractive action of $s \in G$ on $x \in X$ by $s x$, and identifying $L^{1}(G) \widehat{\otimes} X$ with $L^{1}(G, X)$, we see that $(\rho x)(t)=t^{-1} x(x \in X, t \in G)$, defines a linear contraction $\rho$ from $X$ into $L^{1}(G, X)$ such that for all $s \in G$ and $x \in X$,

$$
\rho(s x)(t)=t^{-1}(s x)=\left(s^{-1} t\right)^{-1} x=\left(L_{s} \widehat{\otimes} 1_{X}\right)(\rho x)(t) \quad(t \in G),
$$

and

$$
\pi(\rho x)=\int t(\rho x)(t) d t=\int t\left(t^{-1} x\right) d t=\int x d t=x,
$$

provided the Haar measure of $G$ having been chosen equal to one. This implies the 1-projectivity of $X$.

Here we are rather interested in the converse question: given an $L^{1}(G)$-module $X$, when does the projectivity of $X$ imply the compactness of $G$ ? The main tool for deciding this is the following lemma of Yu. V. Selivanov, cf. [S1, Lemma 1.4, p. 389] and [S2, Corollary 1. p. 212].

1.3. Lemma (Selivanov). Let $A$ be a Banach algebra and $X$ be an essential Banach left $A$-module such that either $A$ or $X$ satisfy Grothendieck's approximation condition. If $X$ is projective then there exists for every $x \neq 0$ in $X$ an A-module homomorphism $\varphi: X \rightarrow A$ with $\varphi(x) \neq 0$.

1.4. Proposition. Let $G$ be a locally compact group. If there exists a projective essential Banach left $L^{1}(G)$-module $X$ with $X^{*}$ being either norm separable or weakly sequentially complete, then $G$ is compact.

Proof. Since $A=L^{1}(G)$ enjoys the approximation property, the projectivity of $X$ implies by (1.3) the existence of a non-zero $L^{1}(G)$-module homomorphism $\varphi: X \rightarrow L^{1}(G)$ such that $\varphi(s x)=L_{s}(\varphi x)$, for all $s \in G, x \in X$. Since the dual map of $\varphi, \varphi^{*}: L^{\infty}(G) \rightarrow X^{*}$, is weakly compact, in the case of $X^{*}$ being norm separable by [G, Corollaire 1, p. 168] and in the case of $X^{*}$ being weakly sequentially complete by [D-S, VI.7.6 Theorem, p. 494], $\varphi$ is weakly compact as well. Since for any $x \in X$ its $G$-orbit $\{s x: s \in G\}$ is norm bounded in $X$, it follows from $\varphi(s x)=L_{s}(\varphi x), s \in G$, that its image is relatively weakly compact in $L^{1}(G)$. Since $\varphi(x) \neq 0$ in $L^{1}(G)$ for some $x \in X$, the Dunford-Pettis theorem implies the compactness of $G$, cf. [La, Theorem 4.8, p. 137] or [R, Lemma 1.1.(a), p. 602]. 
1.5. Example ([D-P Theorem 5.1, p. 415]). Let $X=L^{p}(G), 1<p<\infty$, be endowed with any action making it an essential $L^{1}(G)$-module. Then we have:

$$
L^{p}(G) \text { projective } \Longleftrightarrow G \text { compact }
$$

Let us remark that $L^{1}(G)$ is a projective left $L^{1}(G)$-module for any $G$, by [He1, IV. Theorem 2.17, p. 175].

1.6. ExAmple. Let $\pi$ be a continuous unitary representation of the locally compact group $G$ on a Hilbert space $h$ and let $X=\mathcal{L}^{p}(h), 1<p<\infty$, be the space of all operators $T$ on $h$ such that $\operatorname{trace}\left(T^{*} T\right)^{\frac{p}{2}}<\infty$. Then $X$ is a reflexive Banach space that becomes an essential left $L^{1}(G)$-module under the action $s T=\pi(s) T \pi\left(s^{-1}\right)$, for $s \in G$, $T \in \mathcal{L}^{p}(h)$. Endowing the $C^{*}$-algebra, $K(h)$, of all compact operators on $h$ with the same action and noting that the dual of any $C^{*}$-algebra is weakly sequentially complete, [T1, III. Corollary 5.2, p. 148], we have

$$
K(h), \mathcal{L}^{p}(h) \text { projective } \Longleftrightarrow G \text { compact }
$$

1.7. ExAmple. Let $X$ be either $C^{*}(G)$, the full $C^{*}$-algebra of $G$, or $C_{r}^{*}(G)$, the reduced $C^{*}$-algebra of $G$, endowed with left translation. Then $C^{*}(G)$ and $C_{r}^{*}(G)$ are essential left $L^{1}(G)$-modules whose duals are weakly sequentially complete such that

$$
C^{*}(G), C_{r}^{*}(G) \text { projective } \Longleftrightarrow G \text { compact }
$$

The same applies to the $C^{*}$-algebra, $K\left(L^{2}(G)\right)$, of compact operators on $L^{2}(G)$ with $G$ acting as $s T=L_{s} T L_{s^{-1}}, T \in K\left(L^{2}(G)\right)$, a special case of 1.6.

1.8. ExAmple. Let $X$ be $K\left(L^{p}(G)\right)$ the space of compact operators on $L^{p}(G), 1<$ $p<\infty$. Then $K\left(L^{p}(G)\right)$ is an essential $L^{1}(G)$-module under $s T=L_{s} T L_{s^{-1}}$, whose dual Banach space is isometrically isomorphic to $L^{p}(G) \widehat{\otimes} L^{p^{\prime}}(G)$, which is norm separable whenever the topology of $G$ has a countable base:

$$
K\left(L^{p}(G)\right) \text { projective and } G \text { 2nd-countable } \Longrightarrow G \text { compact }
$$

1.9. Example. Let $A(G)$ be the Fourier algebra of $G$, and $V N(G)$ its von Neumann algebra such that $A(G)^{*}=V N(G)$. If $\varphi$ is a function on $G$ satisfying $\varphi u \in A(G)$ for all $u \in A(G)$, then $\varphi$ is continuous and bounded and defines a bounded linear operator, $m_{\varphi}$, on the Banach space $A(G), m_{\varphi}(u)=\varphi u(u \in A(G))$. With this in mind we define

$$
\begin{aligned}
M A(G) & =\left\{\varphi \in C^{b}(G): \varphi u \in A(G) \quad \forall u \in A(G)\right\}, \\
M_{0} A(G) & =\left\{\varphi \in M A(G):\left(m_{\varphi}\right)^{*}: V N(G) \rightarrow V N(G) \text { completely bounded }\right\},
\end{aligned}
$$

with norms

$$
\begin{aligned}
\|\varphi\|_{M A(G)} & =\left\|m_{\varphi}: A(G) \rightarrow A(G)\right\|, \\
\|\varphi\|_{M_{0} A(G)} & =\left\|\left(m_{\varphi}\right)^{*}: V N(G) \rightarrow V N(G)\right\|_{c b} .
\end{aligned}
$$

$M_{0} A(G)$ is called the space of completely bounded multipliers, and $M A(G)$ the space of all multipliers of $A(G)$. Denoting by $Q_{0}(G)$ and $Q(G)$ the completions of $L^{1}(G)$ with 
respect to the norms

$$
\begin{aligned}
& \|f\|_{Q_{0}(G)}=\sup \left\{\left|\int f(t) \varphi(t) d t\right|: \varphi \in M_{0} A(G),\|\varphi\|_{M_{0} A(G)} \leqslant 1\right\}, \\
& \|f\|_{Q(G)}=\sup \left\{\left|\int f(t) \varphi(t) d t\right|: \varphi \in M A(G),\|\varphi\|_{M A(G)} \leqslant 1\right\} \quad\left(f \in L^{1}(G)\right),
\end{aligned}
$$

we get two translation invariant Banach spaces whose duals are isometrically isomorphic with $M_{0} A(G)$ and $M A(G)$, respectively:

$$
Q_{0}(G)^{*}=M_{0} A(G), \quad Q(G)^{*}=M A(G),
$$

cf. dC-H, 1.10 Proposition, p. 466]. It follows from 1.9 Lemma, p. 465 in [C-H], that $M_{0} A(G)$ and $M A(G)$ are weakly sequentially complete. Since left translation is continuous and isometric on $Q_{0}(G)$ and $Q(G)$, these are essential left $L^{1}(G)$-modules such that we have

$$
Q_{0}(G), Q(G) \text { projective } \Longleftrightarrow G \text { compact }
$$

2. Flatness over $L^{1}(G)$. Rather than giving the original definition, He1, VII. Definition 1.1, p. 239], we shall use the following criterion, due to O. Yu. Aristov [A, Lemma 1.2 , p. 1558], and its dual.

2.1. Criterion (Aristov). Let $A$ be a Banach algebra and $X$ be a Banach left $A$ module. $X$ is flat if and only if there is a bounded linear map $\rho$ from $X$ into the bidual $\left(A_{+} \widehat{\otimes} X\right)^{* *}$ such that $\pi^{* *} \circ \rho=\iota_{X}$, the canonical embedding of $X$ into $X^{* *}$, and $\rho(a x)=$ $\left(L_{a} \widehat{\otimes} 1_{X}\right)^{* *}(\rho x)$, for $x \in X$ and $a \in A$ :

$$
X \stackrel{\rho}{\longrightarrow}\left(A_{+} \widehat{\otimes} X\right)^{* *} \stackrel{\pi^{* *}}{\longrightarrow} X^{* *} .
$$

$X$ is called $c$-flat, for some constant $c>0$, if there is such a $\rho$ of norm $\|\rho\| \leqslant c$, cf. [W], Definition 4.2, p. 164]. If $X$ is essential, $A_{+}$may be replaced by $A$.

2.2. Criterion (dual). Let $A$ be a Banach algebra, $X$ be a Banach left $A$-module and $X^{*}$ its dual right $A$-module. $X$ is flat if and only if there is a bounded linear map $\lambda$ from $\mathcal{L}\left(A_{+}, X^{*}\right)$ into $X^{*}$ such that $\lambda \circ \pi^{*}=1_{X^{*}}$ and $\lambda\left(T \circ L_{a}\right)=(\lambda T) a$, for all $T \in \mathcal{L}\left(A_{+}, X^{*}\right)$ and $a \in X$ :

$$
X^{*} \stackrel{\pi^{*}}{\longrightarrow} \mathcal{L}\left(A_{+}, X^{*}\right) \stackrel{\lambda}{\longrightarrow} X^{*} .
$$

In this case, $X^{*}$ is called an injective right $A$-module, and $c$-injective if there is such a $\lambda$ of norm $\|\lambda\| \leqslant c$. If $X$ is essential, $A_{+}$may again be replaced by $A$.

Clearly, a left $A$-module $X$ is $c$-flat if and only if its dual right $A$-module $X^{*}$ is $c$ injective. For a discussion of injectivity see, for instance, Definition 1.5 and Propositions 1.6 and 1.7 in $[\mathrm{D}-\mathrm{P}$, p. 394].

2.3. Let $A=L^{1}(G)$. If $G$ is amenable then every essential Banach left $L^{1}(G)$-module $X$ is 1-flat. Indeed, let $M$ be a left invariant mean on $L^{\infty}(G)$. Using the isometric isomorphism of $\mathcal{L}\left(L^{1}(G), X^{*}\right)$ with $L_{w^{*}}^{\infty}\left(G, X^{*}\right)$, the space of all bounded functions $\Phi: G \rightarrow X^{*}$ such that, for any $x \in X, t \mapsto\langle x, \Phi(t)\rangle$ is measurable on $G$, there corresponds to every 
$T \in \mathcal{L}\left(L^{1}(G), X^{*}\right)$ a unique function $\Phi \in L_{w^{*}}^{\infty}\left(G, X^{*}\right)$ via the formula

$$
\langle x, T f\rangle=\int_{G} f(t)\langle x, \Phi(t)\rangle d t \quad\left(x \in X, f \in L^{1}(G)\right),
$$

cf. [T1, IV. Proposition 7.16, p. 262]. Considering $X$ as a (continuous, contractive) Banach $G$-module, the function $t \mapsto\left\langle t^{-1} x, \Phi(t)\right\rangle$ is bounded and measurable in $t \in G$ such that

$$
\langle x, \lambda(\Phi)\rangle=\int\left\langle t^{-1} x, \Phi(t)\right\rangle d M(t) \quad\left(x \in X, \Phi \in L_{w^{*}}^{\infty}\left(G, X^{*}\right)\right)
$$

defines a linear contraction $\lambda$,

$$
X^{*} \stackrel{\pi^{*}}{\longrightarrow} L^{\infty}\left(G, X^{*}\right) \stackrel{\lambda}{\longrightarrow} X^{*},
$$

satisfying $\lambda \circ \pi^{*}=1_{X^{*}}$ and $\lambda \circ\left(L_{s} \widehat{\otimes} 1_{X}\right)^{*}(\Phi)=(\lambda \Phi) s$, for all $\Phi \in L_{w^{*}}^{\infty}\left(G, X^{*}\right)$ and $s \in G$. It follows that $X^{*}$ is 1-injective and $X$ 1-flat.

2.4. REMARK. In spite of the similarity of the diagrams in 1.1 and 2.1 one must not expect that every flat Banach left module $X$ over a Banach algebra $A$ admit a non-zero $A$-module homomorphism $\varphi: X \rightarrow A^{* *}$. Indeed, let $G$ be an amenable locally compact group and let $A=L^{1}(G)$ and $X=L^{p}(G), 2<p<\infty$. Then every non-zero left $L^{1}(G)$ module homomorphism $\varphi: L^{p}(G) \rightarrow L^{1}(G)^{* *}$ gives rise to a non-zero left invariant operator $\varphi^{t}: L^{\infty}(G) \rightarrow L^{p^{\prime}}(G)$ which forces $G$ to be compact, cf. [L-vR, Theorem 3, p. 308] or [R, Proposition 1.2, p. 603].

Again, we are interested in the question: given an $L^{1}(G)$-module $X$, when does flatness of $X$ imply amenability of $G$ ? Several examples are given in $[\mathrm{D}-\mathrm{P}$, and we will add a few more.

2.5. ExAmple. Let $X=K\left(L^{p}(G)\right), 1<p<\infty$, be the space of compact operators on $L^{p}(G)$ with the action $s T=L_{s} T L_{s^{-1}}$ for $s \in G, T \in K\left(L^{p}(G)\right)$. Then $K\left(L^{p}(G)\right)$ becomes an essential Banach left $L^{1}(G)$-module whose dual module $L^{p}(G) \widehat{\otimes} L^{p^{\prime}}(G)$ is endowed with the right action $(f \otimes g) s=L_{s^{-1}} f \otimes L_{s-1} g$, for $s \in G$ and $f \otimes g \in L^{p}(G) \widehat{\otimes} L^{p^{\prime}}(G)$. By the left invariance of Haar measure, the duality $\tau: L^{p}(G) \widehat{\otimes} L^{p^{\prime}}(G) \rightarrow \mathbb{C}$ is $G$-invariant such that we infer from Theorem 4.6 in [D-P p. 414]: if $L^{p}(G) \widehat{\otimes} L^{p^{\prime}}(G)$ is injective under the above action then $G$ is amenable. Dually, if $K\left(L^{p}(G)\right)$ is flat then $G$ is amenable, i.e. together with 2.3:

$$
K\left(L^{p}(G)\right) \text { flat } \Longleftrightarrow G \text { amenable }
$$

2.6. EXAmple. Let $\pi$ be a continuous unitary representation of $G$ on the Hilbert space $h, K(h)$ the $C^{*}$-algebra of compact operators on $h$ with $s T=\pi(s) T \pi\left(s^{-1}\right)$, for $s \in G$, $T \in K(h)$, such that its dual module $h \widehat{\otimes} \bar{h}$ has the action $(\xi \otimes \bar{\eta}) s=\pi\left(s^{-1}\right) \xi \otimes \bar{\pi}\left(s^{-1}\right) \bar{\eta}$, for $s \in G$ and $\xi \otimes \bar{\eta} \in h \otimes \bar{h}$ ( $\bar{h}$ and $\bar{\pi}$ denoting the complex-conjugates of $h$ and $\pi$, respectively). Therefore the trace $\tau: h \widehat{\otimes} \bar{h} \rightarrow \mathbb{C}$ is $G$-invariant, and we conclude as in 2.5:

$$
K(h) \text { flat } \Longleftrightarrow G \text { amenable }
$$

2.7. Example. Let $C^{*}(G)$ be the full $C^{*}$-algebra of $G$, and $Q_{0}(G)$ be the Banach space defined in 1.9. Endowing both of them with left translation by $G$, we have 


$$
C^{*}(G), Q_{0}(G) \text { flat } \Longleftrightarrow G \text { amenable }
$$

Proof. One direction follows from 2.3. To prove the other one we shall show that the injectivity of the dual modules, $C^{*}(G)^{*}$ and $Q_{0}(G)^{*}$, implies the amenability of $G$. Identifying $C^{*}(G)^{*}$ with the space, $B(G)$, of coefficients of all continuous unitary representations of $G$, and $Q_{0}(G)^{*}$ with $M_{0} A(G)$, we see that $B(G)$ is contained in $M_{0} A(G)$. By a theorem of Bożejko and Fendler, [B-F] or [J], every $\varphi \in M_{0} A(G)$ can be written as $\varphi\left(t^{-1} s\right)=\left(\Phi_{1}(s) \mid \Phi_{2}(t)\right)$ for $(s, t) \in G \times G$, where $\Phi_{1}, \Phi_{2}: G \rightarrow h$ are two continuous bounded functions with values in some Hilbert space $h$. It follows that every such $\varphi$ is weakly almost periodic: $M_{0} A(G) \subset W A P(G)$. Denoting by $1_{G}$ the constant function corresponding to the trivial representation of dimension one, we have $1_{G} \in B(G) \subset M_{0} A(G) \subset W A P(G)$, and so it suffices to prove the statement for $M_{0} A(G)$.

If $M_{0} A(G)$ is injective as a right Banach $G$-module, we have a map $\lambda$ as in 2.2,

$$
\left.M_{0} A(G) \stackrel{\pi^{*}}{\longrightarrow} \mathcal{L}\left(L^{1}(G), M_{0} A(G)\right) \stackrel{\lambda}{\longrightarrow} M_{0} A(G)\right)
$$

such that $\lambda\left(\pi^{*} \varphi\right)=\varphi$ for $\varphi \in M_{0} A(G)$, and $\lambda\left(T \circ L_{s}\right)=L_{s^{-1}}(\lambda T)$, for $T \in \mathcal{L}\left(L^{1}, M_{0} A\right)$ and $s \in G$. Associating with every $\varphi \in L^{\infty}(G)$ the operator $T_{\varphi}$, as kindly suggested to us by N. Monod, $[\mathrm{M}]$,

$$
T_{\varphi}: L^{1}(G) \rightarrow M_{0} A(G), \quad T_{\varphi}(f)=\langle f, \varphi\rangle 1_{G} \quad\left(f \in L^{1}(G)\right),
$$

we get by left invariance of Haar measure

$$
T_{L_{s} \varphi}(f)=\left\langle f, L_{s} \varphi\right\rangle 1_{G}=\left\langle L_{s^{-1}} f, \varphi\right\rangle 1_{G}=T_{\varphi}\left(L_{s^{-1}} f\right) \quad\left(s \in G, f \in L^{1}(G)\right),
$$

and

$$
T_{1_{G}}(f)=\left\langle f, 1_{G}\right\rangle 1_{G}=1_{G} \otimes 1_{G}(f) \quad\left(f \in L^{1}(G)\right),
$$

such that $T_{1_{G}}=\pi^{*}\left(1_{G}\right)$. Denoting by $m$ the left invariant mean on $W A P(G)$, we see that the composition $M=m \circ \lambda \circ T$ is a non-zero left invariant functional on $L^{\infty}(G)$. Indeed, we have, for any $\varphi \in L^{\infty}(G)$ and $s \in G$,

$$
M\left(L_{s} \varphi\right)=m\left(\lambda\left(T_{L_{s} \varphi}\right)\right)=m\left(\lambda\left(T_{\varphi} \circ L_{s^{-1}}\right)\right)=m\left(L_{s}\left(\lambda\left(T_{\varphi}\right)\right)\right)=m\left(\lambda\left(T_{\varphi}\right)\right)=M(\varphi),
$$

and

$$
M\left(1_{G}\right)=m\left(\lambda\left(T_{1_{G}}\right)\right)=m\left(\lambda\left(\pi^{*}\left(1_{G}\right)\right)\right)=m\left(1_{G}\right)=1,
$$

from which the amenability of $G$ follows.

2.8. In [S2, Theorem 1, p. 211], Selivanov showed that for any projective module $X$ over a Banach algebra $A$ there is a bounded linear projection from $\mathcal{L}(X)$ onto the subspace, $\mathcal{L}_{A}(X)$, of $A$-module homomorphisms. In the same vein, there is for any flat $X$ a bounded linear projection from $\mathcal{L}\left(X^{*}\right)$ onto $\mathcal{L}_{A}\left(X^{*}\right)$, the space of homomorphisms of the dual module $X^{*}$, and if $X$ is $c$-flat the projection can be chosen of norm $\leqslant c$. Since, in this case, $X^{*}$ is injective, this follows from the following lemma which we shall formulate only for left modules.

LEMma. Let $Y$ be a Banach left module over the Banach algebra A. If, for some constant $c>0, Y$ is c-injective, then there is a bounded linear projection, $P$, of norm $\|P\| \leqslant c$ from $\mathcal{L}(Y)$ onto the subspace, $\mathcal{L}_{A}(Y)$, of A-module homomorphisms. 
Proof. According to the definition, cf. [D-P, Proposition 1.6, p. 394], there is a bounded linear map $\lambda$ of norm $\|\lambda\| \leqslant c$, satisfying $\lambda\left(T \circ R_{a}\right)=a(\lambda T)$ and $\lambda(\alpha y)=y$, for $T \in$ $\mathcal{L}\left(A_{+}, Y\right), a \in A$ and $y \in Y$,

$$
Y \stackrel{\alpha}{\longrightarrow} \mathcal{L}\left(A_{+}, Y\right) \stackrel{\lambda}{\longrightarrow} Y,
$$

$\alpha$ being given by $(\alpha y)(a)=a y$, for $y \in Y$ and $a \in A_{+}$, and $R_{a}$ denoting right multiplication by $a$ on $A_{+}$. Defining $P$ by $(P T)(y)=\lambda(T \circ \alpha y)$, for $T \in \mathcal{L}(Y), y \in Y$, we see that $P$ is a bounded linear operator on $\mathcal{L}(Y)$ of norm $\|P\| \leqslant\|\lambda\|$ satisfying, for $T \in \mathcal{L}(Y)$ and $a \in A$,

$$
(P T)(a y)=\lambda(T \circ \alpha(a y))=\lambda\left(T \circ \alpha y \circ R_{a}\right)=a \lambda(T \circ \alpha y)=a(P T)(y),
$$

and, for $T \in \mathcal{L}_{A}(Y)$, in virtue of $T \circ \alpha y=\alpha(T y)$,

$$
(P T)(y)=\lambda(T \circ \alpha y)=\lambda(\alpha(T y))=T y,
$$

such that $P$ is a linear projection from $\mathcal{L}(Y)$ onto $\mathcal{L}_{A}(Y)$ of norm $\|P\| \leqslant c$.

2.9. $A$ von Neumann algebra $\mathcal{M}$ on a Hilbert space $h$ is called injective if there is a linear projection of norm one from $\mathcal{L}(h)$ onto $\mathcal{M}$. By a theorem of Helemskii, He3, Corollary 1 , p. 77], the injectivity of $\mathcal{M}$ implies the injectivity of the Banach left $\mathcal{M}$-module $h$. As a partial converse we have

Corollary. Let $\mathcal{M}$ be a von Neumann algebra on $h$. If the Banach left $\mathcal{M}$-module $h$ is 1-injective, then $\mathcal{M}$ is injective.

Proof. Let the elements of $\mathcal{M}$ act on $h$ as operators. From (2.8), with $A=\mathcal{M}$ and $Y=h$, follows the existence of a linear projection of norm $c=1$ from $\mathcal{L}(h)$ onto $\mathcal{L}_{\mathcal{M}}(h)=\mathcal{M}^{\prime}$, the commutant of $\mathcal{M}$. Hence $\mathcal{M}^{\prime}$ is injective, and so is $\mathcal{M}$, cf. e.g. [T2, XV. Proposition 3.2(iii), p. 174].

REMARK. The question of how the bound of the projection can be relaxed is discussed by Pisier in [P] and by Christensen and Sinclair in [C-S1] and [C-S2].

2.10. ExAmple. Let $G$ be a discrete group and let $l^{1}(G)$ act on $l^{2}(G)$ by left or right convolution. Then the Banach $l^{1}(G)$-module $l^{2}(G)$ is 1-flat if and only if $G$ is amenable:

$$
l^{2}(G) \text { 1-flat } \Longleftrightarrow G \text { amenable }
$$

Proof. Let us consider $l^{2}(G)$ as a right $l^{1}(G)$-module such that $G$ acts on $l^{2}(G)$ by right translation $\left(R_{s} f\right)(t)=f(t s)$, for $s \in G$ and $f \in l^{2}(G)$. If $l^{2}(G)$ is 1-flat, it is 1-injective such that, by 2.8 , there is a projection, $P$, of norm one from $\mathcal{L}\left(l^{2}(G)\right)$ onto $\mathcal{L}_{l^{1}(G)}\left(l^{2}(G)\right)$, the subspace of all operators commuting with $R_{s}, s \in G$, which coincides with the von Neumann algebra, $V N(G)$, generated by the left translation operators $L_{s}, s \in G$. By Tomiyama's Theorem, [T1, III. Theorem 3.4, p. 131], $P$ is actually a $V N(G)$-bimodule homomorphism such that $P\left(L_{s} T L_{s^{-1}}\right)=L_{s}(P T) L_{s^{-1}}$, for all $T \in \mathcal{L}\left(l^{2}(G)\right)$ and $s \in G$. Denoting the multiplication representation of $l^{\infty}(G)$ on $l^{2}(G)$ by $\pi, \pi(\varphi) f=\varphi f$ for $\varphi \in l^{\infty}(G), f \in l^{2}(G)$, and the canonical trace on $V N(G)$ by $\tau, \tau(T)=\left(T \varepsilon_{e} \mid \varepsilon_{e}\right)$ for $T \in V N(G)$, the composition $M=\tau \circ P \circ \pi$ will be a left invariant mean on $L^{\infty}(G)$, as is well known, cf. [Sch, 7. Lemma, p. 23]. The other direction follows, of course, from 2.3. 
3. Questions and remarks. $G$ will denote a locally compact group and $p^{\prime}$ the exponent conjugate to $1<p<\infty$.

3.1. Question (Dales and Polyakov). Let $G$ act by left translation on $L^{p}(G), 1<p<\infty$. Does the flatness of $L^{p}(G)$ as a Banach left module over $L^{1}(G)$ imply the amenability of $G$ ? Or, equivalently, does the injectivity of $L^{p^{\prime}}(G)$ imply the amenability of $G$ ? H. G. Dales and M. E. Polyakov showed in [D-P], Theorem 5.9 and Theorem 5.12, that for no discrete group $G$ containing the free group on two generators $l^{p}(G)$ is injective, and they conjecture that this remains true for all non-amenable discrete groups, D-P, p. 425]. All that is known today is contained in the recent preprint of P. Ramsden [Ra.

3.2. REMARK. Let $G$ be a discrete amenable group acting contractively on a Banach space $X$. If $\lambda: \mathcal{L}\left(L^{1}(G), X^{*}\right) \rightarrow X^{*}$ is the map associated, as in (2.3), with an invariant mean on $G$, then $\lambda(T)$ is contained in the weak *- closed convex hull of the set $\left\{T\left(\varepsilon_{t}\right) \varepsilon_{t^{-1}}\right.$ : $t \in G\}$, for every $T \in \mathcal{L}\left(L^{1}(G), X^{*}\right)$.

Proof. Let $T: L^{1}(G) \rightarrow X^{*}$ be bounded linear and let $\phi: G \rightarrow X^{*}$ be defined by $\phi(t)=T\left(\varepsilon_{t}\right), \varepsilon_{t}$ being the point measure at $t \in G$. If $\lambda$ is associated with the left invariant mean $M$ on $G, 2.3$, we have

$$
\langle x, \lambda T\rangle=\int\left\langle t^{-1} x, \phi(t)\right\rangle d M(t)=\int\left\langle x, \phi(t) t^{-1}\right\rangle d M(t),
$$

for $T \in \mathcal{L}\left(L^{1}(G), X^{*}\right)$ and $x \in X$. If the assertion were wrong there would exist such $T$ and $x$ and two real numbers $\alpha<\beta$ satisfying

$$
\operatorname{Re}\langle x, \lambda T\rangle \leqslant \alpha<\beta \leqslant \operatorname{Re}\left\langle x, \phi(t) t^{-1}\right\rangle \quad(t \in G),
$$

such that averaging with respect to $M$ gives the desired contradiction. (We have written $\left.\phi(t) t^{-1}=T\left(\varepsilon_{t}\right) \varepsilon_{t-1}.\right)$

3.3. Remark. Let $G$ act by left translation on $L^{p}(G), 1<p<\infty$. If $G$ is amenable, but non-compact, then any map $\lambda: \mathcal{L}\left(L^{1}(G), L^{p^{\prime}}(G)\right) \rightarrow L^{p^{\prime}}(G)$ associated with an invariant mean on $G, 2.3$, vanishes on the subspace of compact operators from $L^{1}(G)$ into $L^{p^{\prime}}(G)$. Proof. Since the space of compact operators from $L^{1}(G)$ into $L^{p^{\prime}}(G)$ can be identified with $L^{\infty}(G) \check{\otimes} L^{p^{\prime}}(G)$, the injective tensor product of $L^{\infty}(G)$ with $L^{p^{\prime}}(G)$, and $\lambda$ is linear and continuous, it suffices to show that $\lambda(\varphi \otimes g)=0$ for all $\varphi \in L^{\infty}(G)$ and $g \in L^{p^{\prime}}(G)$. But, for any $f \in L^{p}(G)$, the definition of $\lambda$ associated with the invariant mean $M,(2.3)$ with $x=f$ and $\phi=\varphi \otimes g$, implies

$$
\begin{aligned}
\langle f, \lambda(\varphi \otimes g)\rangle & =\int\left\langle L_{t^{-1}} f, \varphi(t) g\right\rangle d M(t)=\int\left\langle L_{t^{-1}} f, g\right\rangle \varphi(t) d M(t) \\
& =\int g * \check{f}(t) \varphi(t) d M(t)=0,
\end{aligned}
$$

since the convolution $g * \check{f}, \check{f}(t)=f\left(t^{-1}\right)$, vanishes at infinity.

3.4. Denoting by $W A P(G)$ the space of weakly almost periodic functions on $G$ and by $\check{\otimes}$ the injective tensor product of Banach spaces, we have for any $1<p<\infty$ isometric inclusions

$$
C^{o}(G) \check{\otimes} L^{p^{\prime}}(G) \subset W A P(G) \check{\otimes} L^{p^{\prime}}(G) \subset L^{\infty}(G) \check{\otimes} L^{p^{\prime}}(G) \subset L^{\infty}\left(G, L^{p^{\prime}}(G)\right),
$$


the last space being equal to $\mathcal{L}\left(L^{1}(G), L^{p^{\prime}}(G)\right)$, in this case, and $C^{o}(G)$ denoting the space of continuous functions on $G$ vanishing at infinity.

REMARK. Let $G$ be non-compact and $1<p<\infty$. Then any bounded linear map $\lambda$ : $\mathcal{L}\left(L^{1}(G), L^{p^{\prime}}(G)\right) \rightarrow L^{p^{\prime}}(G)$ satisfying $\lambda\left(T \circ L_{s}\right)=L_{s^{-1}}(\lambda T)$, for $T \in \mathcal{L}\left(L^{1}(G), L^{p^{\prime}}(G)\right)$ and $s \in G$, vanishes on the subspace $W A P(G) \check{\otimes} L^{p^{\prime}}(G)$.

Proof. It suffices to show that $\lambda(\varphi \otimes g)=0$ for all $\varphi \in W A P(G)$ and $g \in L^{p^{\prime}}(G)$. For any fixed $g \in L^{p^{\prime}}(G)$, we consider the bounded linear operator $\lambda_{1}$,

$$
\lambda_{1}: L^{\infty}(G) \rightarrow L^{p^{\prime}}(G), \quad \lambda_{1}(\varphi)=\lambda(\varphi \otimes g) \quad\left(\varphi \in L^{\infty}(G)\right),
$$

satisfying $\lambda_{1}\left(L_{s} \varphi\right)=L_{s}\left(\lambda_{1} \varphi\right), s \in G$ and $\varphi \in L^{\infty}(G)$, because of

$$
\lambda_{1}\left(L_{s} \varphi\right)=\lambda\left(L_{s} \varphi \otimes g\right)=\lambda\left(\varphi \otimes g \circ L_{s^{-1}}\right)=L_{s} \lambda(\varphi \otimes g)=L_{s}\left(\lambda_{1} \varphi\right) .
$$

Let $\varphi \in W A P(G)$. The set $\left\{L_{s} \varphi: s \in G\right\}$ being relatively weakly compact in $L^{\infty}(G)$, we obtain in virtue of the Dunford-Pettis property of $L^{\infty}(G),[\mathrm{G}$, Proposition 1, p. 135, and Théorème 1(a), p. 139], and the weak compactness of $\lambda_{1}$, that the $\operatorname{set}\left\{\lambda_{1}\left(L_{s} \varphi\right)\right.$ : $s \in G\}=\left\{L_{s}\left(\lambda_{1} \varphi\right): s \in G\right\}$ is relatively norm compact in $L^{p^{\prime}}(G)$, implying $\lambda_{1} \varphi=0$, by [La, Theorem 4.6, p. 136] or [R, Lemma 1.1.(b), p. 602].

3.5. Remark. Let $G$ be non-compact and $2<p<\infty$. Then any bounded linear map $\lambda: \mathcal{L}\left(L^{1}(G), L^{p^{\prime}}(G)\right) \rightarrow L^{p^{\prime}}(G)$ satisfying $\lambda\left(T \circ L_{s}\right)=L_{s^{-1}}(\lambda T), T \in \mathcal{L}\left(L^{1}(G), L^{p^{\prime}}(G)\right)$ and $s \in G$, vanishes on the subspace of all compact operators from $L^{1}(G)$ into $L^{p^{\prime}}(G)$.

Proof. For any fixed $g \in L^{p^{\prime}}(G)$, let $\lambda_{1}: L^{\infty}(G) \rightarrow L^{p^{\prime}}(G), \lambda_{1}(\varphi)=\lambda(\varphi \otimes g), \varphi \in L^{\infty}(G)$, be the left invariant operator considered in the proof of (3.4). Since $1<p^{\prime}<2$, it follows from [L-vR, Theorem 3, p. 308], that $\lambda_{1}=0$ such that $\lambda(\varphi \otimes g)=0$ for all $\varphi \in L^{\infty}(G)$ and $g \in L^{p^{\prime}}(G)$, implying the assertion.

3.6. Question (Gordin). Let $G$ act by left translation on $C_{r}^{*}(G)$, the reduced $C^{*}$-algebra of $G$. Does the flatness of $C_{r}^{*}(G)$ as a Banach left module over $L^{1}(G)$ imply the amenability of $G$ ? - This question, related to $(2.7)$, is due to M. Gordin, Go]. The proof for $C^{*}(G)$ in $(2.7)$ does not apply directly since the constant function $1_{G}$ is in $\left(C_{r}^{*}(G)\right)^{*}$ if and only if $G$ is amenable.

3.7. Question. Let $G$ act by left translation on $Q(G)$, the predual of $M A(G)$ described in (1.9). Does the flatness of $Q(G)$ as a Banach left module over $L^{1}(G)$ imply the amenability of $G$ ? - The proof for $Q_{0}(G)$, as given in (2.7), does not apply since the dual $Q(G)^{*}=M A(G)$ may contain functions which are not weakly almost peroidic.

3.8. Question. Let $\mathcal{M}$ be a von Neumann algebra on the Hilbert space $h$. By a theorem of A.Ya. Helemskii, [He3, Theorem, p. 77], the injectivity of the von Neumann algebra $\mathcal{M}$ implies the injectivity of any normal dual Banach module over the Banach algebra $\mathcal{M}$. Is any such module already 1-injective in the sense of (2.2)? To be more explicit, let $\mathcal{M}$ be injective, $X$ be a Banach left $\mathcal{M}$-module with dual right module $X^{*}$ such that, for all $\left(x, x^{*}\right) \in X \times X^{*}$, the linear form $a \mapsto\left\langle a x, x^{*}\right\rangle, a \in \mathcal{M}$, is $\sigma$-weakly continuous on $\mathcal{M}$. Does there exist a linear map $\lambda$ satisfying $\lambda\left(T \circ L_{a}\right)=(\lambda T) a$, for $T \in \mathcal{L}\left(\mathcal{M}, X^{*}\right), a \in \mathcal{M}$, 
and being left inverse to $\pi^{*},\left(\pi^{*} x^{*}\right)(a)=x^{*} a$, for $x^{*} \in X^{*}, a \in \mathcal{M}$,

$$
X^{*} \stackrel{\pi^{*}}{\longrightarrow} \mathcal{L}\left(\mathcal{M}, X^{*}\right) \stackrel{\lambda}{\longrightarrow} X^{*}
$$

such that $\|\lambda\|=1$ ?

Note added in proof. The answer to 3.8 seems to be yes; cf. the forthcoming paper "On injective von Neumann algebras", to appear in Proc. Amer. Math. Soc.

Acknowledgements. It is a pleasure to thank the Erwin Schrödinger International Institute for Mathematical Physics, ESI, in Vienna for its hospitality during the workshop "Amenability on Groups and Beyond" and, in particular, M. Gordin and N. Monod for their suggestions and questions.

This paper is based on a lecture delivered at the $19^{\text {th }}$ International Conference on Banach Algebras held at Będlewo, July 14-24, 2009. The support for the meeting by the Polish Academy of Sciences, the European Science Foundation under the ESF-EMSERCOM partnership, and the Faculty of Mathematics and Computer Science of the Adam Mickiewicz University at Poznań is gratefully acknowledged.

\section{References}

[A] O. Yu. Aristov, On approximation of flat Banach modules by free modules, Matem. Sbornik 196 (2005), 3-32 (Russian); Sbornik: Mathem. 196 (2005), 1553-1583 (English).

[B-F] M. Bożejko and G. Fendler, Herz-Schur multipliers and completely bounded multipliers of the Fourier algebra of a locally compact group, Boll. Un. Mat. Ital. (6) 3-A (1984), 297-302.

[C-S1] E. Christensen and A. M. Sinclair, On von Neumann algebras which are complemented subspaces of $B(H)$, J. Funct. Anal. 122 (1994), 91-102.

[C-S2] E. Christensen and A. M. Sinclair, Module mappings into von Neumann algebras and injectivity, Proc. London Math. Soc. (3) 71 (1995), 618-640.

[D-P] H. G. Dales and M. E. Polyakov, Homological properties of modules over group algebras, Proc. London Math. Soc. (3) 89 (2004), 390-426.

[dC-H] J. deCannière and U. Haagerup, Multipliers of the Fourier algebras of some simple Lie groups and their discrete subgroups, Amer. J. Math. 107 (1985), 455-500.

[D-S] N. Dunford and J. T. Schwartz, Linear Operators. Part I, Interscience Publishers, New York, 1957.

[G] A. Grothendieck, Sur les applications linéaires faiblement compactes d'espaces du type $C(K)$, Can. J. Math. 5 (1953), 129-173.

[Go] M. Gordin, Private communication, ESI, Vienna, 2007.

[He1] A. Ya. Helemskii, The homology of Banach and topological algebras, Izdat. Moskow. Gos. Univ., Moscow, 1986; English transl. Kluwer, Dordrecht, 1989.

[He2] A. Ya. Helemskii, The homological essence of Connes amenability: Injectivity of the predual bimodule, Matem. Sbornik 180 (1989), 1680-1690 (Russian); Math. USSR Sbornik 68 (1991), 555-566 (English).

[He3] A. Ya. Helemskii, The spatial flatness and injectiveness of Connes operator algebras, Extracta Math. 9 (1994), 75-81. 
[J] P. Jolissaint, A characterization of completely bounded multipliers of Fourier algebras, Colloq. Math. 63 (1992), 311-313.

[La] A. T.-M. Lau, Closed convex invariant subsets of $L_{p}(G)$, Trans. Amer. Math. Soc. 232 (1977), 131-142.

[L-vR] T.-S. Liu and A. C. M. van Rooij, Translation invariant maps $L^{\infty}(G) \rightarrow L^{p}(G)$, Indag. Mathem. 36 (1974), 306-316.

[M] N. Monod, Private communication, ESI, Vienna, 2007.

[P] G. Pisier, Projections from a von Neumann algebra onto a subalgebra, Bull. Soc. Math. France 123 (1995), 139-153.

[R] G. Racher, Some remarks on a paper by Liu and van Rooij, Indag. Mathem. 18 (2007), 601-609.

[Ra] P. Ramsden, Multi-norms and modules over group algebras, Preprint, 2009.

[Sch] J. Schwartz, Two finite, non-hyperfinite, non-isomorphic factors, Comm. Pure Appl. Math. 16 (1963), 19-26.

[S1] Yu. V. Selivanov, Biprojective Banach algebras, Izv. Akad. Nauk SSR, Ser. Mat. 43 (1979), 1159-1174 (Russian); Math. USSR Izvestiya 15 (1980), 387-399 (English).

[S2] Yu. V. Selivanov, Projective Fréchet modules with the approximation property, Uspekhi Matem. Nauk 50 (1995), 209-210 (Russian); Russian Math. Surveys 50 (1995), 211-213 (English).

[T1] M. Takesaki, Theory of Operator Algebras I, Springer-Verlag, New York, 1979.

[T2] M. Takesaki, Theory of Operator Algebras III, Springer-Verlag, Berlin, 2002.

[W] M. C. White, Injective modules for uniform algebras, Proc. London Math. Soc. (3) 73 (1996), 155-184. 
